\title{
Electron Energy Loss Spectroscopy Measurement of the Optical Gaps on Individual Boron Nitride Single-Walled and Multiwalled Nanotubes
}

\author{
R. Arenal, ${ }^{1}$ O. Stéphan, ${ }^{2}$ M. Kociak,${ }^{2}$ D. Taverna, ${ }^{2}$ A. Loiseau, ${ }^{1}$ and C. Colliex ${ }^{2}$ \\ ${ }^{1}$ Laboratoire d'Etude des Microstructures, UMR 104 ONERA-CNRS, ONERA B P72, 92322 Châtillon Cedex, France \\ ${ }^{2}$ Laboratoire de Physique Solides, CNRS, UMR8502, Université Paris-Sud, 91405 Orsay, France
}

(Received 15 January 2005; published 16 September 2005)

\begin{abstract}
Spatially resolved electron energy loss spectroscopy experiments have been performed in an electron microscope on several individual boron nitride (BN) single-, double-, and triple-walled nanotubes, whose diameters and number of shells have been carefully measured. In the low-loss region (from 2 to $50 \mathrm{eV}$ ) the spectra have been analyzed within the framework of the continuum dielectric theory, leading to the conclusion of a weak influence of out-of-plane contribution to the dielectric response of the tubes. The gap has been measured to be independent of the nanotubes geometry, and close to the in-plane gap value of hexagonal $\mathrm{BN}(5.8 \pm 0.2 \mathrm{eV})$.
\end{abstract}

DOI: 10.1103/PhysRevLett.95.127601

PACS numbers: 79.20.Uv, 73.22.-f, 78.67.Ch

The synthesis of several types of nanotubes: carbon (C), boron nitride $(\mathrm{BN})$, and tungsten disulphide $\left(\mathrm{WS}_{2}\right)$, and other inorganic materials [1] has provided a fertile field for both experimental and complementary theoretical studies. Shortly after the predictions on the stability of BN tubes $[2,3]$, their synthesis was achieved in the form of multiwalled nanotubes (MWNTs) [4-6]. More recently, singlewalled nanotubes (SWNTs) have been made available [7]. Pure BN tubes (BNNTs) have attracted increasing attention based on the theoretically predicted mechanical stability, chemical inertness and electronic insulating behavior independent of chirality and number of tube walls $[2,3]$.

As most of the predicted properties (electronic, optical) are dependent on the value of the band gap, it is of highest importance to validate (or not) the theoretical studies $[2,3]$, which have predicted band gap values rather insensitive to the detailed BNNT structure for experimentally available diameters and close to that for hexagonal boron nitride (h-BN). Very little is experimentally known on the electronic properties of BNNTs and the performed measurements to date concern assemblies of BNNTs, which have not been characterized individually. Transport measurements on small BNNT ropes [8] have shown that, as expected, all nanotubes are semiconductors [3]. Optical absorption spectroscopy on assemblies of the same BNSWNTs has not provided band-gap measurements but has identified lines located more than $1 \mathrm{eV}$ below the band gap of h-BN [9], which have been attributed to Frenkel excitons. On the other hand, STM/STS studies have been performed on BN-MWNTs with large diameters (5 to $20 \mathrm{~nm}$ ) [10]. The electronic band gap was determined to vary from tube to tube, and to be of the order of $4.6 \mathrm{eV}$, which is smaller than the predicted values.

To remedy to this lack of accurate and reliable experimental data, we present in this Letter spatially resolved electron energy loss spectroscopy (EELS) experiments on several single-walled (SWNT), double-walled (DWNT), and triple-walled (TWNT) BNNT. After separating spa- tially the surface and bulk contributions, the results are analyzed within the framework of the continuum dielectric theory. It provides us with an innovative method to estimate the optical gap for individual nanotubes selfsupported in vacuum, whose radii and number of shells are determined in parallel. The gap values are found to be independent of the nanotube geometry, and close to the gap value of the h-BN $(5.8 \pm 0.2 \mathrm{eV})$.

The present EELS measurements covering an energy loss range up to $30 \mathrm{eV}$ have been performed in a scanning transmission electron microscope (STEM) focusing a sub$\mathrm{nm}$ incident electron probe on the investigated nanotubes. Previous low-loss TEM-EELS studies have been published by Terauchi [11] and our group [12]. In the latter study, the gap region was not addressed, while in the former one, the probe area was much larger than the investigated structure, and therefore the discrimination between surface and bulk effects, as well as the identification of anisotropy effects were not possible. This is also the case in Ref. [13], where the electronic structure of a macroscopic number of $\mathrm{BN}$ MWNT of various diameters and chiralities has been measured by EELS.

For a bulk material, the optical gap is generally measured by optical absorption spectroscopy, providing with a measure of $\epsilon_{2}$, the imaginary part of the dielectric function $\epsilon$. EELS on a bulk specimen is well known to measure the loss function: $\operatorname{Im}(-1 / \epsilon)$. The gap value can therefore be measured as the onset of the loss function, and $\epsilon_{2}$ extracted through a Kramers-Kronig analysis [14].

For individual nanotubes the previous description fails because the surface electromagnetic response is highly dominant. In such a case, the electromagnetic response can be dealt with when a fast electron beam passes near the considered nano-object without intersecting it ("aloof spectroscopy" [15]), and excites only surface modes. For a cylindrical object perpendicular to the beam axis, the EELS spectrum is proportional to a weighted sum of multipolar polarizabilities $\alpha_{m}(q)$, with weights decreasing rap- 
idly as the azimuthal momentum $m$ and the wave vector along the axis, $q$, increase [16]. Thus, in a good approximation, the surface EELS spectra are proportional to $\operatorname{Im}\left(\alpha_{0}(q \rightarrow 0)\right)$, namely, the optical polarizability. When the beam intersects the nanotube, the situation is more intricate, but we have shown on carbon nanotubes that essentially a (small) "volume" contribution adds up to the surface spectra [17].

Among several descriptions, the continuum dielectric theory $[15,18]$ has demonstrated great success to both enlighten the physics of the electromagnetic response of nanoparticles and provide analytical formula for computing polarizabilities of different geometries. This model assumes that the response of a nanoparticle to an electromagnetic field is locally described by the macroscopic dielectric tensor of the corresponding bulk material - the $\mathrm{h}$-BN in the case of BN tubes. We have shown experimentally and theoretically $[17,19]$ that, for MWNTs for which the ratio between inner and outer radii tends to 1 , and a fortiori for SWNT, the polarizabilities are proportional to $\operatorname{Im}\left(-1 / \epsilon_{\|}(\omega)+\epsilon_{\perp}(\omega)\right)$, where $\epsilon_{\|}(\omega)$ and $\epsilon_{\perp}(\omega)$ are, respectively, the components of the dielectric tensor parallel and perpendicular to the anisotropy axis, and $\omega$ is the energy. A direct measurement of a quantity proportional to the optical absorption perpendicular to the anisotropy axis can thus be obtained, providing direct information on gaps and interband energy values. One of the purposes of this work is to use this experimental approach and extend the above model for measuring optical gaps of individual nano-objects.

Synthesis of BN-SWNT has been achieved as described in Ref. [7] via laser vaporization of a h-BN target. A typical sample only contains a moderate fraction of nanotubes (SWNT, and MWNT) amongst different by-products such as h-BN platelets, BN shells encapsulating a boron core, and different boron based compounds. The complexity of the sample further advocates for using spatially resolved spectroscopic methods.

Experiments were performed on the Orsay VG HB501 STEM delivering a $0.5 \mathrm{~nm}$ diameter electron probe. EELS spectra were recorded with a home made 2D CCD camera optically coupled to a scintillator in the imaging plane of a Gatan 666 spectrometer. The spectral information was basically acquired in two modes. In cases where a high signal to noise ratio (SNR) was aimed, the beam was rastered on a nanoarea $(2 \mathrm{~nm} \times 3 \mathrm{~nm})$ centered on the nanotube. In that case, the spectrum is an average spectrum displaying excitations from both penetrating and nonpenetrating geometries. In order to retrieve the spatial dependence of the electromagnetic excitations, the experiments were also performed in the spectrum-line mode (see Fig. 1). In this mode, the electron probe is scanned from the vacuum across the nanotube, a spectrum being recorded at each point (or impact parameter) of the scan. The High Angle Annular Dark Field signal, directly proportional to

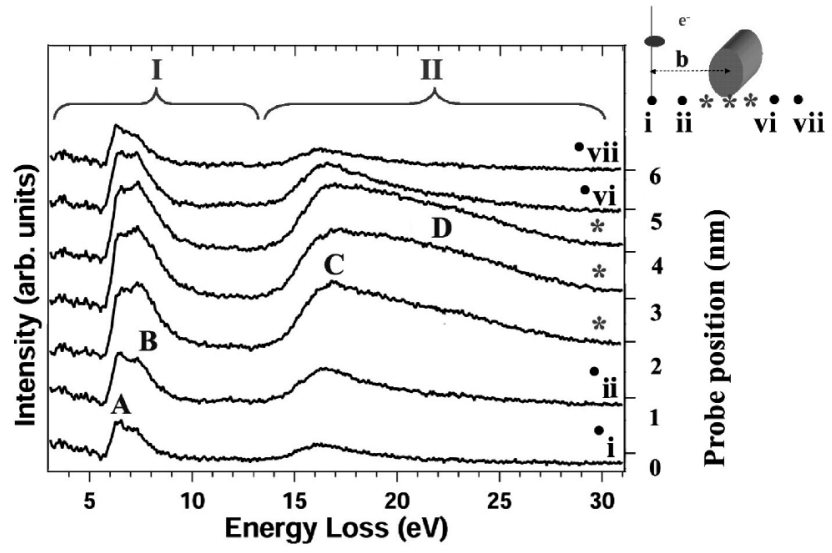

FIG. 1. A series of deconvoluted spectra when the electron beam is moved across a BN TWNT, as sketched in the inset. Different spectral features are indicated by letters (A to D), and the two main excitation bands by numbers I and II.

the projected mass under the beam, is also recorded during the scan. It allows to unambiguously separate the "surface" and volume origins of the different features in the EELS spectra. Before and after each experiment, a STEM bright field image was acquired for a careful estimation of the structural parameters of the tubes (number of walls and diameter) and to check that no tube damaging nor contamination has biased the acquired spectra.

As the main goal of this work is to measure the gap of individual nanotubes around $5 \mathrm{eV}$, the best energy resolution is required and the contribution of the zero-loss (ZL) tail, that generally blinds the gap energy region, has to be reduced at most. We have thus systematically recorded series (10 to 500) of spectra with a short acquisition time (typically $50 \mathrm{~ms}$ ), which are subsequently realigned and summed and then processed with a deconvolution procedure based on maximum likelihood algorithm [20]. This increases the energy resolution down to about $0.2 \mathrm{eV}$. Finally, a self-deconvoluted ZL acquired in the vacuum is subtracted from the original deconvoluted spectra, which provides a very flat profile of quasinull intensity in the band-gap region.

The results obtained on a total population of 23 SWNTs, 3 DWNTs, and 4 TWNTs can be summarized as follows. Figure 1 shows a sequence of deconvoluted energy loss spectra acquired while scanning the $0.5 \mathrm{~nm}$ probe step by step, perpendicular to the axis of a triple-wall BNNT. The penetrating (and nonpenetrating) regions are emphasized. In both cases, one clearly identifies two energy bands, the first one being usually interpreted as arising from $\pi-\pi^{*}$ excitations (labeled I), while the second one is usually related to $\pi-\sigma^{*}$ and $\sigma-\sigma^{*}$ excitations (labeled II). As seen in Fig. 1, the onset of the excitations, which corresponds, as stated above, to the optical gap, does not shift during the scan. The gap can therefore be directly measured on rastered-mode spectra, for which a high SNR is available. Moreover, the overall spectrum shape is only 
slightly modified between inside and outside the tube in the case of the MWNT: one notices a clear relative increase of peak $B$ versus peak $A$ in the penetrating positions, and the appearance of a small bump (D) around $20 \mathrm{eV}$. These two peaks are related to bulk excitations of presumably plasmonic nature. They will not be further detailed and interpreted. In the case of SWNTs (not shown here), any change is hardly detectable.

To further detail the different excitation modes, Fig. 2 displays a collection of spectra acquired on different nanotubes, either in the rastered mode over a SWNT $(2.2 \mathrm{~nm}$ in diameter) or using a probe fixed either at grazing incidence or over the tubes for a DWNT and a TWNT ( 2.8 and $3 \mathrm{~nm}$ of external diameter, respectively). For comparison, a spectrum acquired on a thin foil of h-BN with the anisotropic axis set parallel to the penetrating electron trajectory is also shown. In this figure, we have also introduced the $\epsilon_{\perp, 2}$ contribution deduced by a Kramers-Kronig transformation from the h-BN energy loss function. It is worth mentioning the close similarity of this curve and the response of the nanotubes. Also, the similarities between the SWNT spectrum and those of the DWNT and the TWNT acquired in the near-field geometry are striking. In particular, the positions of the different energy peaks are, within $0.2 \mathrm{eV}$, identical. Referring to Fig. 1, region I is constituted by a sharp and intense first peak at $6.5 \mathrm{eV}$ (labeled A) followed by a shoulder at $7.5 \mathrm{eV}$ (labeled B), while region II displays one broad peak (labeled C) centered on $16-17 \mathrm{eV}$.

We now turn to the interpretation of the different surface excitation features, within the above recalled continuum dielectric description. The multipolar polarizabilities $\alpha_{m}(q)$ are computed analytically with the bulk dielectric tensor $\bar{\epsilon}(\omega)$ as an input parameter [16]. Because of the large discrepancies found in the literature concerning the

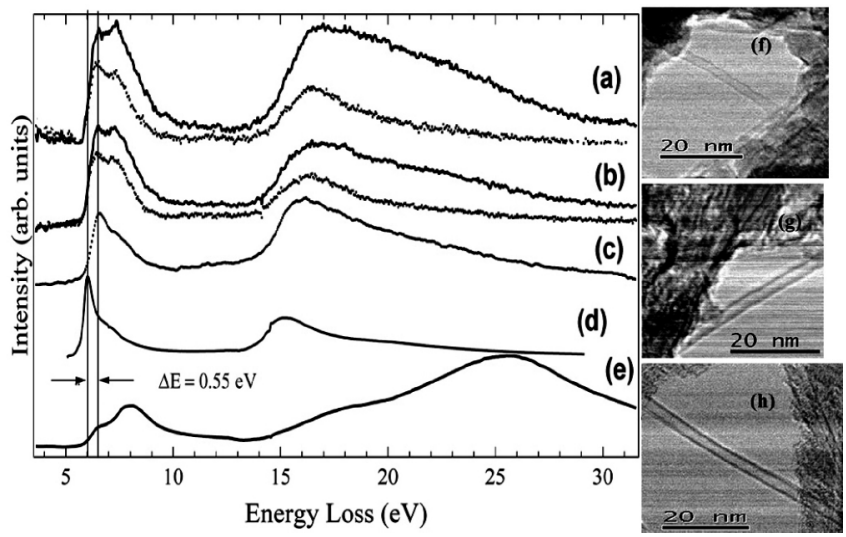

FIG. 2. From top to bottom: Experimental deconvoluted spectra for: (a) a TWNT, inside the tube and at grazing incidence, (b) a DWNT inside the tube and at grazing incidence, (c) a SWNT in the rastered mode, (d) $\epsilon_{\perp, 2}$ as extracted from the bulk loss spectra in (e). (f), (g), (h): Bright field images of the tubes (a), (b), (c). dielectric constant of $\mathrm{h}-\mathrm{BN}$, we have used a dielectric constant computed with the $a b$ initio CASTEP code (Accelrys $\operatorname{Inc}$.). $\operatorname{Im}\left(\epsilon_{\perp}(\omega)\right)$ and $\operatorname{Im}\left(-1 / \epsilon_{\|}(\omega)\right)$ curves deduced from these coefficients are displayed in Fig. 3 (bottom). The EELS spectra for given impact parameters are then calculated. Figure 3 (top) shows the results for grazing incidence on the SWNT and the TWNT experimentally studied in Fig. 2. The agreement is rather good, except for the absence in the model of the shoulder at $7.5 \mathrm{eV}$ (feature B of Fig. 1) which is generally attributed to an interband transition, and a rigid shift of the energy positions. They both likely find their origin in the deficiencies of the dielectric constants calculated with CASTEP. Furthermore, the enhancement of peak B in Fig. 1 when the probe is positioned on top of thicker tubes also suggests the contribution of a bulk type effect, which the present description does not account for. Quite remarkably, the similarity between theoretical EELS and calculated $\epsilon_{\perp, 2}(\omega)$ curves reproduces that observed between experimental EELS and measured $\epsilon_{\perp, 2}(\omega)$ curves. It also implies that the contribution of the out-of-plane excitation [see curve $\operatorname{Im}\left(-1 / \epsilon_{\|}(\omega)\right)$ in Fig. 3] remains quite reduced. In both cases, the $0.4-0.5 \mathrm{eV}$ shift of peak a and the $0.8 \mathrm{eV}$ shift of peak $\mathrm{C}$ from Fig. 1 to higher energy between $\epsilon_{\perp, 2}(\omega)$ and EELS spectra for SWNT and MWNT are well reproduced.

We have previously shown $[16,17,19]$ that unperfect electromagnetic coupling between surfaces of a nanotube affects the positions of the observed maxima in the spectrum. This is the cause of the discrepancies (slight shifts) between the spectra and corresponding $\epsilon_{\perp, 2}(\omega)$. At the opposite - and this is a capital point — we have checked that these coupling effects do not affect the position of the gap. This gives a second justification that the optical gap can readily be measured on such near-field EELS spectra.

As the present simulation, which uses a homogeneous dielectric theory, correctly reproduces the experimental spectra for curved structures with different numbers of

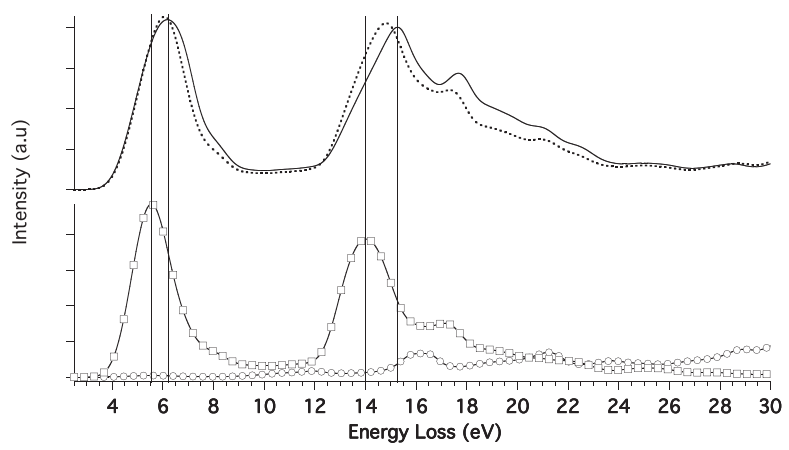

FIG. 3. Top: Simulation of grazing incidence spectra for a SWNT (dashed line) and a TWNT (solid line) of radii equal to those of Fig. 2. Bottom: $\epsilon_{\perp, 2}(\omega)$ (squares) and $\operatorname{Im}\left(-1 / \epsilon_{\|}(\omega)\right)$ (circles) computed with the dielectric tensor component of the h-BN used for the above simulations. 


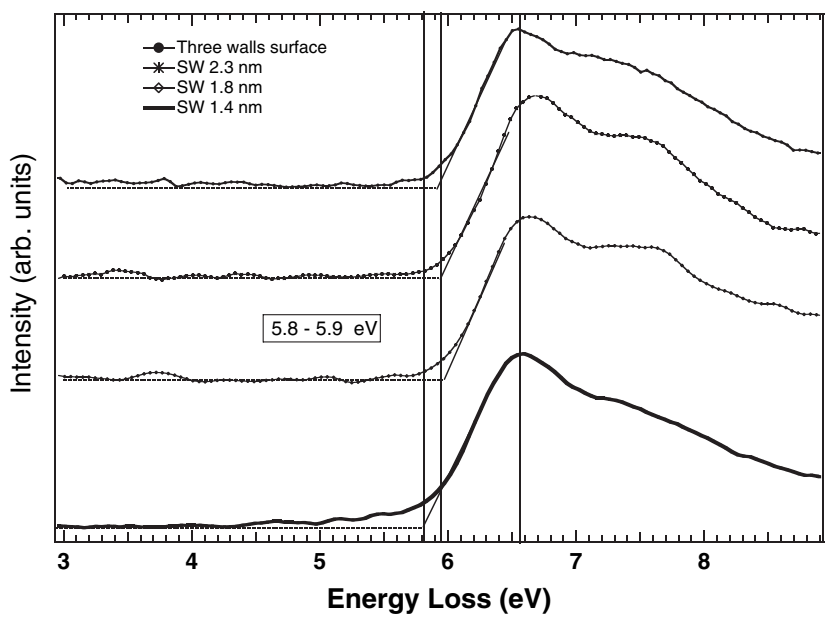

FIG. 4. Gap measurement for (top to bottom) a TWNT (grazing incidence) and three SWNT.

layers, one may question why the results are so weakly dependent on the atomic structure. Our results first show that the major part of the response is contained within the layers, so that the influence of the interaction between neighboring layers remains quite weak. As for the curvature effects which could introduce some partial hybridization with $s p_{3}$ orbitals, it has been shown in the case of graphitic nanotubes that it could only be observable when the radius of curvature is smaller than $0.5 \mathrm{~nm}$ [21]. The BNNT analyzed here all exhibit larger radii.

Following the above confirmation that EELS spectra recorded at grazing incidence reflect the absorption spectra and that the coupling effects between inner and outer surfaces do not affect the position of the gap, the optical gap in individual nanotubes is measured at the intersection between the flat background and the line fitting the slope of the first peak (labeled A in Fig. 1) of the spectra, as shown in Fig. 4. For all investigated nanotubes (diameter ranging from 1.5 to $3 \mathrm{~nm}$ and number of layers from 1 to 3 ), the value of the optical gap is found to be $5.8 \pm 0.2 \mathrm{eV}$, which is very close to that of bulk h-BN. If the precise value of the gap may depend on the specific procedure and definition used to measure it, its constant value between tubes does not. This very small dispersion in the gap values and the similarity with h-BN [14] confirms the ab initio predictions [3]. It also raises the interest and advantage of measuring individual objects with simultaneous control of their structure as compared to measurements on macroscopic populations of nano-objects.

We have presented the first experimental study of optical properties of individual BNNT of known structures, including SWNT. It is also the first use of EELS aloof spectroscopy to determine the optical band gap. The methodology used is of quite general interest and could be used to investigate band gaps of individual nanostructures over a large spectral domain, from the visible up to the VUV range. The onset energy of optical excitations has been shown to remain constant, independent of the number of layers or the radii of the tubes, and to have a value of $5.8 \pm$ $0.2 \mathrm{eV}$, which is very close to that of the bulk h-BN. The good agreement between the experimental results and the calculations within the classical continuum dielectric theory leads us to conclude that: (1) The optical response is dominated by in-plane excitations. (2) It is not experimentally affected by the interlayer coupling, nor by the curvature. In order to refine the interpretation of the fine structures and to further test the field of application of the dielectric theory, further $a b$ initio calculations are required. They should also confirm, or refute, the absence of lowdimensional quantum effects revealed by the present study.

The authors are grateful to A. Gloter, L. Reining, L. Wirtz, A. Rubio, J. S. Lauret, Ph. Lambin, L. Henrard, and J.L. Cochon for helpful discussions and assistance. This work was supported by the French grant "Naboco," by the European RTN contracts "COMELCAN" (No. HPRN-CT-2000-00128) and "FULLMAT" (No. HPRN-CT-2002-00209), and by the GDR-E NanoE (No. 2756).

[1] Carbon Nanotubes: Synthesis, Structure, Properties, and Applications, edited by P.A.M.S. Dresselhaus and G. Dresselhaus (Springer-Verlag, Berlin, 2001).

[2] A. Rubio, J. L. Corkill, and M. L. Cohen, Phys. Rev. B 49, 5081 (1994).

[3] X. Blase, A. Rubio, S. G. Louie, and M. L. Cohen, Europhys. Lett. 28, 335 (1994).

[4] N. G. Chopra et al., Science 269, 966 (1995).

[5] A. Loiseau, F. Willaime, N. D. G. Hug, and H. Pascard, Phys. Rev. Lett. 76, 4737 (1996).

[6] D. Golberg et al., Appl. Phys. Lett. 69, 2045 (1996).

[7] R. S. Lee et al., Phys. Rev. B 64, 121405 (2001).

[8] M. Radosavljevic et al., Appl. Phys. Lett. 82, 4131 (2003).

[9] J. Lauret et al., Phys. Rev. Lett. 94, 037405 (2005).

[10] R. Czerw et al., Appl. Phys. Lett. 83, 1617 (2003).

[11] M. Terauchi, M. Tanaka, T. Matsumoto, and Y. Saito, J. Electron Microsc. 47, 319 (1998).

[12] M. Kociak, L. Henrard, O. Stéphan, K. Suenaga, and C. Colliex, Phys. Rev. B 61, 13936 (2000).

[13] G. Fuentes et al., Phys. Rev. B 67, 35429 (2003).

[14] C. Tarrio and S. Schnatterly, Phys. Rev. B 40, 7852 (1989).

[15] T. L. Ferrell and P. M. Echenique, Phys. Rev. Lett. 55, 1526 (1985).

[16] D. Taverna, M. Kociak, V. Charbois, and L. Henrard, Phys. Rev. B 66, 235419 (2002).

[17] O. Stéphan et al., Phys. Rev. B 66, 155422 (2002).

[18] L. Henrard and P. Lambin, J. Phys. B 29, 5127 (1996).

[19] M. Kociak et al., Phys. Rev. Lett. 87, 075501 (2001).

[20] A. Gloter, A. Douiri, M. Tence, and C. Colliex, Ultramicroscopy 96, 385 (2003).

[21] K. Suenaga et al., Phys. Rev. B 63165408 (2001). 